\title{
Biohydrogen Production from Waste Biomass
}

\author{
Ming-Jun Zhu* and Huan-Na Lin \\ School of Bioscience \& Bioengineering, South China University of Technology, Guangzhou Higher \\ Education Mega Center, China
}

Received April 8, 2016; Published April 13, 2016

Biohydrogen production from waste biomass has recently gained more attention. At the South China University of Technology, our research group focuses on biohydrogen production from waste biomass that is based on a consolidated bioprocessing strategy. Cassava pulp, paper sludge, sugarcane bagasse, and spent mushroom compost have all been demonstrated to be feasible feedstocks for hydrogen production with Clostridium thermocellum. Furthermore, co-culture of $C$. thermocellum and Thermoanaerobacterium aotearoense can enhance the hydrogen production [1]. Besides, our studies showed that surfactant PEG8000 and $\mathrm{CaCO}_{3}$ both have positive effects on the hydrogen production by C. thermocellum [2-4].

How does biohydrogen production from waste biomass catch our eyes and attract us to devote our efforts on it? With the rapid development of economy, all kinds of waste biomass produced every year are increasing. Recycle of waste biomass can avoid pollution following unsatisfactory disposal such as discard, landfill, and incineration in the field directly. Moreover, waste biomass as raw materials have no conflict with food supply. Thus, there is no doubt that biohydrogen from waste biomass is a promising environmentally friendly energy carrier for its high energy content, and its burning feature of producing only water as the by-product. Biohydrogen from waste biomass is renewable and has no contribution to environmental pollution and climate change, which are valuable for the current situation of increasingly serious energy and environmental issues in China and all over the world. Bioprocesses for hydrogen production from a variety of biomass materials even waste biomass are very promising in the near future.

It is wonderful to produce biohydrogen from waste biomass, however, there are some barriers blocking us out of industrial production. Though the utilization of abundant waste biomass can lower the feedstock cost, the cost of biohydrogen production is still less competitive over traditional fuel oil. Furthermore, an inexpensive and simple pretreatment of waste biomass is needed for biohydrogen production. Technological barriers like hydrogen storage, compressor and distribution networks, lack of durable fuel cell technologies, and integration with the existing infrastructure remain to be overcome [5]. The significant problem of low hydrogen yield could be solved by metabolic engineering or co-culture of microorganisms with different advantages. Ideal biohydrogen production should meet the demand of high hydrogen yield and productivity simultaneously. 
Appropriate bioreactor design, hydrogen production in non-sterile conditions, and feasible techniques for separation or purification of hydrogen are beneficial to practical application. It is important to take the application requirement into consideration, when we are busy in studying biological technologies to improve the hydrogen yield. Advances in optimized processes and technologies would bring us closer to industrially viable biohydrogen production from waste biomass. A comprehensive consideration on industrial feasibility and laboratory investigation would accelerate the large scale production of biohydrogen.

\section{REFERENCES}

[1] Cheng, J., Zhu, M. A novel anaerobic co-culture system for bio-hydrogen production from sugarcane bagasse. Bioresource Technology, 2013, 144: 623-631. DOI:

10.1016/j.biortech.2013.07.018

[2] Cheng, J., Yu, Y., Zhu, M. Enhanced biodegradation of sugarcane bagasse by

Clostridium thermocellum with surfactant addition. Green Chemistry, 2014, 16(5): 26892695. DOI: 10.1039/C3GC42494D

[3] Tian, Q.Q., Liang, L., Zhu, M.J. Enhanced biohydrogen production from sugarcane bagasse by Clostridium thermocellum supplemented with $\mathrm{CaCO}_{3}$, Bioresource Technology, 2015, 197: 422-428. DOI: 10.1016/j.biortech.2015.08.111

[4] Lin, H.N., Hu, B.B., Zhu, M.J. Enhanced hydrogen production and sugar accumulation from spent mushroom compost by Clostridium thermocellum supplemented with PEG8000 and JFC-E, International Journal of Hydrogen Energy, 2016, 41(4): 23832390. DOI: $10.1016 /$ j.ijhydene.2015.11.078

[5] Kumar, G.R., Chowdhary, N. Biotechnological and bioinformatics approaches for augmentation of biohydrogen production: A review. Renewable and Sustainable Energy Reviews, 2016, 56: 1194-1206. DOI: 10.1016/j.rser.2015.12.022

Article copyright: (C) 2016 Ming-Jun Zhu and Huan-Na Lin. This is an open access article distributed under the terms of the Creative Commons Attribution 4.0 International License, which permits unrestricted use and distribution provided the original author and source are credited. 